\title{
A REMARK ON H-CONTACT UNIT TANGENT SPHERE BUNDLES
}

\author{
Sun Hyang Chun, Hong Kyung Pak, Jeong Hyeong Park, \\ AND Kouei SEKIGAWA
}

\begin{abstract}
We shall give some curvature conditions for the unit tangent sphere bundle of an $n(\geq 4)$-dimensional Riemannian manifold to be $\mathrm{H}$ contact. Furthermore, we provide an example illustrating Main Theorem.
\end{abstract}

\section{Introduction}

Studying the relationships between the geometric structures of Riemannian manifolds and their respective unit tangent sphere bundles is one of interesting topics in Riemannian geometry. A unit vector field $V$ on $M$ determines a map between $(M, g)$ and $\left(T_{1} M, g^{\prime}\right)$. If the Riemannian manifold $(M, g)$ is compact and orientable, the energy of $V$ is defined as the energy of the corresponding map:

$$
E(V)=\frac{1}{2} \int_{M}|d V|^{2} d v_{g}=\frac{m}{2} \operatorname{vol}(M, g)+\frac{1}{2} \int_{M}|\nabla V|^{2} d v_{g}
$$

where $m=\operatorname{dim} M$ [14]. $V$ is said to be a harmonic vector field if it is a critical point for the energy functional $E$ in the set of all unit vector fields of $M$ [14].

Perrone defined an $\mathrm{H}$-contact manifold as a contact metric manifold whose characteristic vector field $\xi$ is harmonic, and proved that a contact metric manifold is an $\mathrm{H}$-contact manifold if and only if the characteristic vector field $\xi$ is an eigenvector of the Ricci operator [13]. Boeckx and Vanhecke [3] proved that the unit tangent sphere bundle of a 2-dimensional or 3-dimensional Riemannian manifold is H-contact if and only if the base manifold has constant sectional curvature. Calvaruso and Perrone [5] obtained the same result in the case of the $n(\geq 4)$-dimensional conformally flat manifold. The authors proved that the unit tangent sphere bundle $T_{1} M$ of an $n(\geq 3)$-dimensional Einstein manifold is $\mathrm{H}$-contact if and only if the base manifold is 2-stein ([8], Main Theorem). The main purpose of the present paper is to prove the following:

Received October 23, 2009; Revised February 24, 2010.

2010 Mathematics Subject Classification. Primary 53C25, 53D10.

Key words and phrases. unit tangent sphere bundle, H-contact manifold. 
Main Theorem. Let $M=(M, g)$ be an $n(\geq 2)$-dimensional Riemannian manifold whose unit tangent sphere bundle $T_{1} M$ equipped with the standard contact metric structure $(\eta, \bar{g}, \phi, \xi)$ is $H$-contact. If $\operatorname{dim} M \neq 4$, then the scalar curvature $\tau$ of $M$, the square norm $|\rho|^{2}$ of the Ricci tensor and the square norm $|R|^{2}$ of the curvature tensor are all constant. If $\operatorname{dim} M=4$, then $\tau$ and $|\rho|^{2}$ are constant, however, $|R|^{2}$ is not necessary constant.

This Main Theorem together with Theorem 2 in Section 5 can be comparable with the results ([7], Theorem 1 and Theorem 2). After the proof of Main Theorem, we shall provide an example concerning Main Theorem.

The authors would like to express their thanks to the referee for the insightful suggestion concerning Question 1.

\section{Standard contact metric structure on a unit tangent sphere bundle}

All manifolds in this paper are assumed to be of class $C^{\infty}$. We refer to [2] for the basic concepts and terminologies on contact metric manifolds.

Let $(M, g)$ be an $n$-dimensional Riemannian manifold and $\nabla$ the associated Levi Civita connection. Its Riemann curvature tensor $R$ is defined by $R(X, Y) Z=\nabla_{X} \nabla_{Y} Z-\nabla_{Y} \nabla_{X} Z-\nabla_{[X, Y]} Z$ for all vector fields $X, Y$ and $Z$ on $M$. The tangent bundle of $(M, g)$ is denoted by $T M$ and consists of pairs $(p, u)$, where $p$ is a point in $M$ and $u$ a tangent vector to $M$ at $p$. The mapping $\pi: T M \rightarrow M, \pi(p, u)=p$ is the natural projection from $T M$ onto $M$. For a vector field $X$ on $M$, its vertical lift $X^{v}$ on $T M$ is the vector field defined by $X^{v} \omega=\omega(X) \circ \pi$, where $\omega$ is a 1-form on $M$. For a Levi Civita connection $\nabla$ on $M$, the horizontal lift $X^{h}$ of $X$ is defined by $X^{h} \omega=\nabla_{X} \omega$. The tangent bundle $T M$ can be endowed in a natural way with a Riemannian metric $\tilde{g}$, the so-called Sasaki metric, depending only on the Riemannian metric $g$ on $M$. It is determined by

$$
\tilde{g}\left(X^{h}, Y^{h}\right)=\tilde{g}\left(X^{v}, Y^{v}\right)=g(X, Y) \circ \pi, \quad \tilde{g}\left(X^{h}, Y^{v}\right)=0
$$

for all vector fields $X$ and $Y$ on $M$. Also, $T M$ admits an almost complex structure tensor $J$ defined by $J X^{h}=X^{v}$ and $J X^{v}=-X^{h}$. Then $\tilde{g}$ is a Hermitian metric for the almost complex structure $J$.

The unit tangent sphere bundle $\bar{\pi}: T_{1} M \rightarrow M$ is a hypersurface of $T M$ given by $g_{p}(u, u)=1$. Note that $\bar{\pi}=\pi \circ i$, where $i$ is the immersion. A unit normal vector field $N=u^{v}$ to $T_{1} M$ is given by the vertical lift of $u$ for $(p, u)$. The horizontal lift of a vector is tangent to $T_{1} M$, but the vertical lift of a vector is not tangent to $T_{1} M$ in general. So, we define the tangential lift of $X$ to $(p, u) \in T_{1} M$ by

$$
X_{(p, u)}^{t}=(X-g(X, u) u)^{v} .
$$

Clearly, the tangent space $T_{(p, u)} T_{1} M$ is spanned by vectors of the form $X^{h}$ and $X^{t}$, where $X \in T_{p} M$. 
We now define the standard contact metric structure of the unit tangent sphere bundle $T_{1} M$ of a Riemannian manifold $(M, g)$. The metric $g^{\prime}$ on $T_{1} M$ is induced from the Sasaki metric $\tilde{g}$ on $T M$. Using the almost complex structure $J$ on $T M$, we define a unit vector field $\xi^{\prime}$, a 1 -form $\eta^{\prime}$ and a $(1,1)$-tensor field $\phi^{\prime}$ on $T_{1} M$ by

$$
\xi^{\prime}=-J N, \quad \phi^{\prime}=J-\eta^{\prime} \otimes N .
$$

Since $g^{\prime}\left(\bar{X}, \phi^{\prime} \bar{Y}\right)=2 d \eta^{\prime}(\bar{X}, \bar{Y}),\left(\eta^{\prime}, g^{\prime}, \phi^{\prime}, \xi^{\prime}\right)$ is not a contact metric structure. If we rescale by

$$
\xi=2 \xi^{\prime}, \quad \eta=\frac{1}{2} \eta^{\prime}, \quad \phi=\phi^{\prime}, \quad \bar{g}=\frac{1}{4} g^{\prime},
$$

we get the standard contact metric structure $(\eta, \bar{g}, \phi, \xi)$. From now on, we consider $T_{1} M=\left(T_{1} M, \eta, \bar{g}, \phi, \xi\right)$ with the standard contact metric structure.

Let $\left\{e_{1}, \ldots, e_{n}=u\right\}$ be an orthonormal basis of $T_{p} M$. Then the Ricci tensor $\bar{\rho}$ of $T_{1} M$ is given by

$$
\begin{aligned}
\bar{\rho}\left(X^{t}, Y^{t}\right)= & (n-2)(g(X, Y)-g(X, u) g(Y, u)) \\
& +\frac{1}{4} \sum_{i=1}^{n} g\left(R(u, X) e_{i}, R(u, Y) e_{i}\right), \\
\bar{\rho}\left(X^{t}, Y^{h}\right)= & \frac{1}{2}\left(\left(\nabla_{u} \rho\right)(X, Y)-\left(\nabla_{X} \rho\right)(u, Y)\right), \\
\bar{\rho}\left(X^{h}, Y^{h}\right)= & \rho(X, Y)-\frac{1}{2} \sum_{i=1}^{n} g\left(R\left(u, e_{i}\right) X, R\left(u, e_{i}\right) Y\right),
\end{aligned}
$$

where $\rho$ denotes the Ricci curvature tensor of $M$. We refer to $[4,7,11,12]$ for the formula (2.1).

\section{H-contact unit tangent sphere bundles}

Let $M=(M, g)$ be an $n(\geq 3)$-dimensional Riemannian manifold and $\left\{e_{i}\right\}_{i=1}^{n}$ be a local orthonormal frame field at an arbitrary point $p \in M$. Now, we assume that $T_{1} M$ is $\mathrm{H}$-contact with respect to the standard contact metric structure $(\eta, \bar{g}, \phi, \xi)$. Then the base manifold $M$ satisfies the following conditions [5].

$$
\begin{gathered}
\nabla_{i} \rho_{j k}-\nabla_{j} \rho_{i k}=0, \\
2 \rho_{a b}=\sum_{i, j=1}^{n} R_{a i b j} R_{a i a j} \quad(a \neq b) .
\end{gathered}
$$

From (3.1), we see easily that the scalar curvature $\tau$ of $M$ is constant. Now, we shall deduce several easy consequences of formula (3.2) for the later use. We set

$$
\left\{\begin{array}{l}
u=\cos \theta e_{a}+\sin \theta e_{b}, \\
x=-\sin \theta e_{a}+\cos \theta e_{b} \quad \text { for all } a \neq b
\end{array}\right.
$$


Substituting (3.3) into the left hand side of (3.2), we get (using some standard trigonometric identities)

(3.4) $2 \rho\left(\cos \theta e_{a}+\sin \theta e_{b},-\sin \theta e_{a}+\cos \theta e_{b}\right)=2 \rho_{a b} \cos (2 \theta)+\left(\rho_{b b}-\rho_{a a}\right) \sin (2 \theta)$.

Similarly, substituting (3.3) into the right hand side of (3.2), and taking account of $(3.3)$, we get

$$
\begin{gathered}
\sum_{i, j=1}^{n} R\left(\cos \theta e_{a}+\sin \theta e_{b}, e_{i},-\sin \theta e_{a}+\cos \theta e_{b}, e_{j}\right) \\
\times R\left(\cos \theta e_{a}+\sin \theta e_{b}, e_{i}, \cos \theta e_{a}+\sin \theta e_{b}, e_{j}\right) \\
=2 \rho_{a b} \cos (2 \theta)+\frac{1}{4}\left\{\sum_{i, j=1}^{n}\left(R_{b i b j}\right)^{2}-\sum_{i, j=1}^{n}\left(R_{a i a j}\right)^{2}\right\} \sin (2 \theta) \\
+\frac{1}{4}\left\{\sum_{i, j=1}^{n}\left(R_{a i b j}\right)^{2}+\sum_{i, j=1}^{n} R_{a i b j} R_{b i a j}+\sum_{i, j=1}^{n} R_{a i a j} R_{b i b j}\right. \\
\left.-\frac{1}{2} \sum_{i, j=1}^{n}\left(R_{a i a j}\right)^{2}-\frac{1}{2} \sum_{i, j=1}^{n}\left(R_{b i b j}\right)^{2}\right\} \sin (4 \theta) .
\end{gathered}
$$

Then, comparing the finite Fourier series in (3.4) and (3.5), we obtain two equations:

$$
\begin{gathered}
4\left(\rho_{a a}-\rho_{b b}\right)=\sum_{i, j=1}^{n}\left(R_{a i a j}\right)^{2}-\sum_{i, j=1}^{n}\left(R_{b i b j}\right)^{2} \\
2\left\{\sum_{i, j=1}^{n}\left(R_{a i b j}\right)^{2}+\sum_{i, j=1}^{n} R_{a i b j} R_{b i a j}+\sum_{i, j=1}^{n} R_{a i a j} R_{b i b j}\right\} \\
=\sum_{i, j=1}^{n}\left(R_{a i a j}\right)^{2}+\sum_{i, j=1}^{n}\left(R_{b i b j}\right)^{2} .
\end{gathered}
$$

We shall recall the following fact ([8], Lemma 4.1) which plays an important role in the proof of Main Theorem.

Lemma 1. Let $S^{n}(n \geq 2)$ be an $n$-dimensional unit sphere centered at the origin 0 in an $(n+1)$-dimensional Euclidean space $\mathbb{E}^{n+1}$ and $f$ be a real-valued function on $S^{n}$ satisfying the condition $f(u)=f(v)$ for any $u, v \in S^{n}$ such that $u \perp v$. Then, $f$ is constant on $S^{n}$.

\section{Proof of Main Theorem}

Let $M=(M, g)$ be an $n(\geq 3)$-dimensional Riemannian manifold satisfying the hypothesis of Main Theorem and $\left\{e_{i}\right\}_{i=1}^{n}$ be any orthonormal basis of $T_{p} M$ 
at an arbitrary point $p \in M$. Then from the equality (3.6), we get

$$
4 \rho_{a a}-\sum_{i, j=1}^{n}\left(R_{a i a j}\right)^{2}=4 \rho_{b b}-\sum_{i, j=1}^{n}\left(R_{b i b j}\right)^{2}
$$

for all $a \neq b$. We may regard $\left(T_{p} M, g_{p}\right)$ as an $n$-dimensional Euclidean space $\mathbb{E}^{n}$ and the unit tangent sphere $U_{p}=\left\{u \in T_{p} M|| u \mid=1\right\}$ as an $(n-1)$-dimensional unit sphere $S^{n-1}\left(\subset \mathbb{E}^{n}\right)$, respectively. We now consider the smooth function $F$ on $\mathbb{E}^{n}=\left(T_{p} M, g_{p}\right)$ defined by

$$
F(u)=4 \rho(u, u) g(u, u)-\sum_{i, j=1}^{n}\left(R_{u i u j}\right)^{2}
$$

for all $u \in T_{p} M$. Further, we denote by $f$ the restriction of the function $F$ to $S^{n-1}=U_{p}$. Then, applying Lemma 1 to the function $f$ on $S^{n-1}$, we see that there exists a function $C$ on $M$ satisfying the following equality for any $u \in S^{n-1}$, at each point $p \in M$ :

$$
4 \rho(u, u)-\sum_{i, j=1}^{n}\left(R_{u i u j}\right)^{2}=C(p) .
$$

From (4.3), we have also

$$
4 \rho(u, u) g(u, u)-\sum_{i, j=1}^{n}\left(R_{u i u j}\right)^{2}=C(p) g(u, u) g(u, u)
$$

for any $u \in T_{p} M=\mathbb{E}^{n}$, at each point $p \in M$. We set $u=\sum_{i=1}^{n} u_{i} e_{i}$. Then, from (4.4), we have

$$
\begin{aligned}
& \sum_{a, b, c, d=1}^{n}\left\{\sum_{(a, b, c, d) \in \mathfrak{S}_{4}}\left(4 \rho_{a b} g_{c d}-\sum_{i, j=1}^{n} R_{a i b j} R_{c i d j}\right)\right\} u_{a} u_{b} u_{c} u_{d} \\
= & C(p) \sum_{a, b, c, d=1}^{n}\left\{\sum_{(a, b, c, d) \in \mathfrak{S}_{4}} g_{a b} g_{c d}\right\} u_{a} u_{b} u_{c} u_{d}
\end{aligned}
$$

for any $\left(u_{i}\right) \in \mathbb{E}^{n}$, where $\mathfrak{S}_{4}$ denotes the set of all permutations of the letters $a, b, c, d$. Since we may regard both sides of the equality (4.5) as homogeneous symmetric polynomials of degree 4 , from (4.5), we have

$$
\sum_{(a, b, c, d) \in \mathfrak{S}_{4}}\left(4 \rho_{a b} g_{c d}-\sum_{i, j=1}^{n} R_{a i b j} R_{c i d j}\right)=C(p) \sum_{(a, b, c, d) \in \mathfrak{S}_{4}} g_{a b} g_{c d}
$$


at each point $p \in M$. From (4.6), by direct calculations, we get

$$
\begin{gathered}
4\left(\rho_{a b} g_{c d}+\rho_{a c} g_{b d}+\rho_{a d} g_{b c}+\rho_{b c} g_{a d}+\rho_{b d} g_{a c}+\rho_{c d} g_{a b}\right) \\
-\sum_{i, j=1}^{n}\left(R_{a i b j} R_{c i d j}+R_{a i b j} R_{d i c j}+R_{a i c j} R_{b i d j}+R_{a i c j} R_{d i b j}\right. \\
\left.\quad+R_{a i d j} R_{b i c j}+R_{a i d j} R_{c i b j}\right) \\
=2 C(p)\left(g_{a b} g_{c d}+g_{a c} g_{b d}+g_{a d} g_{b c}\right)
\end{gathered}
$$

for any $1 \leq a, b, c, d \leq n$. Transvecting $g_{c d}$ with (4.7), we have

$$
4(n+4) \rho_{a b}+4 \tau g_{a b}+2 \sum_{i, j=1}^{n} \rho_{i j} R_{a i b j}-3 \sum_{i, j, k=1}^{n} R_{k i a j} R_{k i b j}=2(n+2) C(p) g_{a b} .
$$

From (4.8), we have immediately

$$
\begin{aligned}
2 n(n+2) C(p) & =4(n+4) \tau+4 n \tau-2|\rho|^{2}-3|R|^{2} \\
& =8(n+2) \tau-2|\rho|^{2}-3|R|^{2}
\end{aligned}
$$

From (4.9), we may see that $C$ gives rise to a smooth function on $M$. Thus, from (4.9), since $\tau$ is constant, we have

$$
2 n(n+2) u C=-2 u|\rho|^{2}-3 u|R|^{2}
$$

for any tangent vector $u$ at any point $p \in M$. Now, since $\tau$ is constant, from (4.8), taking account of the second Bianchi identity and (3.1), we have

$$
2 \sum_{a, i, j=1}^{n}\left(\nabla_{a} \rho_{i j}\right) R_{a i b j}-3 \sum_{a, i, j, k=1}^{n} R_{k i a j}\left(\nabla_{a} R_{k i b j}\right)=2(n+2) \nabla_{b} C,
$$

and hence,

$$
-\frac{3}{4} \nabla_{b}|R|^{2}=2(n+2) \nabla_{b} C
$$

Thus, from (4.11), we have

$$
-3 u|R|^{2}=8(n+2) u C .
$$

Thus, from (4.10) and (4.12), we have

$$
8 u|\rho|^{2}+3(4-n) u|R|^{2}=0
$$

for any tangent vector $u$ at each point $p \in M$. Therefore, if $n=4$, then $|\rho|^{2}$ is constant. 
On the other hand, operating $\nabla_{a}$ on both sides of (4.7) and taking sum with respect to $a$, we have

$$
\begin{gathered}
4\left(\nabla_{d} \rho_{b c}+\nabla_{c} \rho_{b d}+\nabla_{b} \rho_{c d}\right) \\
-\sum_{i, j=1}^{n}\left(R_{a i b j} \nabla_{a} R_{c i d j}+R_{a i b j} \nabla_{a} R_{d i c j}+R_{a i c j} \nabla_{a} R_{b i d j}\right. \\
\left.\quad+R_{a i c j} \nabla_{a} R_{d i b j}+R_{a i d j} \nabla_{a} R_{b i c j}+R_{a i d j} \nabla_{a} R_{c i b j}\right) \\
=2\left(\left(\nabla_{b} C\right) g_{c d}+\left(\nabla_{c} C\right) g_{b d}+\left(\nabla_{d} C\right) g_{b c}\right) .
\end{gathered}
$$

Here, we get

$$
\begin{aligned}
\sum_{a, i, j=1}^{n} R_{a i b j} \nabla_{a} R_{c i d j} & =\frac{1}{2} \sum_{a, i, j=1}^{n} R_{a i b j}\left(\nabla_{a} R_{c i d j}-\nabla_{i} R_{c a d j}\right) \\
& =-\frac{1}{2} \sum_{a, i, j=1}^{n} R_{a i b j} \nabla_{c} R_{i a d j} \\
& =\frac{1}{2} \sum_{a, i, j=1}^{n} R_{a i b j} \nabla_{c} R_{a i d j} .
\end{aligned}
$$

Similarly, we have the following.

$$
\begin{aligned}
& \sum_{a, i, j=1}^{n} R_{a i b j} \nabla_{a} R_{d i c j}=\frac{1}{2} \sum_{a, i, j=1}^{n} R_{a i b j} \nabla_{d} R_{a i c j}, \\
& \sum_{a, i, j=1}^{n} R_{a i c j} \nabla_{a} R_{b i d j}=\frac{1}{2} \sum_{a, i, j=1}^{n} R_{a i c j} \nabla_{b} R_{a i d j}, \\
& \sum_{a, i, j=1}^{n} R_{a i c j} \nabla_{a} R_{d i b j}=\frac{1}{2} \sum_{a, i, j=1}^{n} R_{a i c j} \nabla_{d} R_{a i b j}, \\
& \sum_{a, i, j=1}^{n} R_{a i d j} \nabla_{a} R_{b i c j}=\frac{1}{2} \sum_{a, i, j=1}^{n} R_{a i d j} \nabla_{b} R_{a i c j}, \\
& \sum_{a, i, j=1}^{n} R_{a i d j} \nabla_{a} R_{c i b j}=\frac{1}{2} \sum_{a, i, j=1}^{n} R_{a i d j} \nabla_{c} R_{a i b j} .
\end{aligned}
$$

Thus, from (4.15) and (4.16), transvecting $\left(\nabla_{b} C\right) g_{c d}+\left(\nabla_{c} C\right) g_{b d}+\left(\nabla_{d} C\right) g_{b c}$ with the left hand side of (4.14), we have

$$
\begin{gathered}
\left.4 \sum_{-\frac{1}{2} \sum}\left(\left(\nabla_{b} C\right) g_{c d}+\left(\nabla_{c} C\right) g_{b d} C\right) g_{c d}+\left(\nabla_{c} C\right) g_{b d}+\left(\nabla_{d} C\right) g_{b c}\right) \times\left(\nabla_{d} \rho_{b c}+\nabla_{c} \rho_{b d}+\nabla_{b} \rho_{c d}\right) \\
\times\left(\nabla_{b}\left(R_{a i c j} R_{a i d j}\right)+\nabla_{c}\left(R_{a i b j} R_{a i d j}\right)+\nabla_{d}\left(R_{a i b j} R_{a i c j}\right)\right) .
\end{gathered}
$$


Similarly, transvecting $\left(\nabla_{b} C\right) g_{c d}+\left(\nabla_{c} C\right) g_{b d}+\left(\nabla_{d} C\right) g_{b c}$ with the right hand side of (4.14), we have

$$
\begin{aligned}
& 2 \sum\left(\left(\nabla_{b} C\right) g_{c d}+\left(\nabla_{c} C\right) g_{b d}+\left(\nabla_{d} C\right) g_{b c}\right) \\
& \times\left(\left(\nabla_{b} C\right) g_{c d}+\left(\nabla_{c} C\right) g_{b d}+\left(\nabla_{d} C\right) g_{b c}\right) \\
&=2 \sum\left\{4\left(\nabla_{b} C\right)^{2}+4\left(\nabla_{c} C\right)^{2}+4\left(\nabla_{d} C\right)^{2}+\left(\nabla_{b} C\right)^{2}+\left(\nabla_{d} C\right)^{2}\right. \\
&\left.+\left(\nabla_{c} C\right)^{2}+\left(\nabla_{d} C\right)^{2}+\left(\nabla_{b} C\right)^{2}+\left(\nabla_{c} C\right)^{2}\right\} \\
&=36|\nabla C|^{2},
\end{aligned}
$$

and hence, from (4.17) and (4.18), we get

$$
\begin{aligned}
36|\nabla C|^{2} & \\
=-\frac{1}{2} & \left\{2 \sum\left(\nabla_{b} C\right)\left(\nabla_{c}\left(R_{a i b j} R_{a i c j}\right)\right)+\sum\left(\nabla_{b} C\right)\left(\nabla_{b}|R|^{2}\right)\right. \\
& +2 \sum\left(\nabla_{c} C\right)\left(\nabla_{b}\left(R_{a i c j} R_{a i b j}\right)\right)+\sum\left(\nabla_{c} C\right)\left(\nabla_{c}|R|^{2}\right) \\
& \left.+2 \sum\left(\nabla_{d} C\right)\left(\nabla_{b}\left(R_{a c b j} R_{a i d j}\right)\right)+\sum\left(\nabla_{d} C\right)\left(\nabla_{d}|R|^{2}\right)\right\} \\
=- & \frac{9}{4} \sum\left(\nabla_{i} C\right) \nabla_{i}|R|^{2} .
\end{aligned}
$$

Thus, we have

$$
16|\nabla C|^{2}=-\sum\left(\nabla_{i} C\right) \nabla_{i}|R|^{2} .
$$

From (4.11) and (4.19), we have

$$
128(n+2)|\nabla C|^{2}=\left.\left.3|\nabla| R\right|^{2}\right|^{2} .
$$

On one hand, from (4.11), we have

$$
64(n+2)^{2}|\nabla C|^{2}=\left.\left.9|\nabla| R\right|^{2}\right|^{2} .
$$

Thus, from (4.21) and (4.20), we have

$$
\left.\left.6|\nabla| R\right|^{2}\right|^{2}=\left.\left.(n+2)|\nabla| R\right|^{2}\right|^{2},
$$

and hence,

$$
\left.\left.(n-4)|\nabla| R\right|^{2}\right|^{2}=0 .
$$

Thus, if $n \neq 4$, then $\nabla|R|^{2}=0$, and hence, $|R|^{2}$ is a constant. Therefore, from (4.13), it follows that $|\rho|^{2}$ is a constant. This completes the proof of Main Theorem.

Here, we remark that an $\eta$-Einstein structure is a special case of an $\mathrm{H}$ contact. In [7], we proved that if $T_{1} M$ is $\eta$-Einstein, then $\tau,|R|^{2}$ and $|\rho|^{2}$ are all constants.

Lastly, we provide an example illustrating the latter part of Main Theorem. 
Let $M$ be a 4-dimensional real half-space given by $M=\left\{\left(x_{1}, x_{2}, x_{3}, x_{4}\right) \in\right.$ $\left.\mathbb{R}^{4} \mid x_{1}>0,\left(x_{2}, x_{3}, x_{4}\right) \in \mathbb{R}^{3}\right\}$ and define a Riemannian metric $g$ on $M$ by

$$
g=\left(g_{i j}\right)=\left(\begin{array}{cccc}
x_{1} & 0 & 0 & 0 \\
0 & x_{1}+\frac{x_{3}^{2}}{4 x_{1}} & -\frac{x_{2} x_{3}}{4 x_{1}} & \frac{x_{3}}{2 x_{1}} \\
0 & -\frac{x_{2} x_{3}}{4 x_{1}} & x_{1}+\frac{x_{2}^{2}}{4 x_{1}} & -\frac{x_{2}}{2 x_{1}} \\
0 & \frac{x_{3}}{2 x_{1}} & -\frac{x_{2}}{2 x_{1}} & \frac{1}{x_{1}}
\end{array}\right),
$$

where $g_{i j}=g\left(\frac{\partial}{\partial x_{i}}, \frac{\partial}{\partial x_{j}}\right)[9,10]$.

Then we see that $(M, g)$ is Ricci flat and 2-stein, and hence, the unit tangent sphere bundle $T_{1} M$ equipped with the standard contact metric structure is $\mathrm{H}$ contact. However, we may also check that the square norm of the curvature tensor is not constant [8]. Reflecting on Main Theorem, the following question will naturally arise.

Question 1. Is an $n(\neq 4)$-dimensional Riemannian manifold, whose unit tangent sphere bundle equipped with the standard contact metric structure having $\mathrm{H}$-contact, locally symmetric?

\section{An application}

In this section, we shall provide an application concerning Main Theorem. First, we define symmetric $(0,2)$-tensor field $\alpha$ on $M$ by

$$
\alpha(x, y)=\sum_{i, j, k} R_{x i j k} R_{y i j k}
$$

for any $x, y \in T_{p} M$ at each point $p \in M$. An $n$-dimensional Einstein manifold $M=(M, g)$ is called a super-Einstein manifold [4] if $M$ additionally satisfies the condition

$$
\alpha=\frac{|R|^{2}}{n} g
$$

We here remark that the constancy of $|R|^{2}$ follows from the condition (5.2) for an $n(\neq 4)$-dimensional super-Einstein manifold ([4], Lemma 3.3). For a 4dimensional super-Einstein manifold, the constancy of $|R|^{2}$ is usually required ([4], p. 531). We may easily check that a 2-stein manifold satisfies the condition (5.2). It is also well-known that every harmonic space is super-Einstein [1].

For the remainder of this section, we assume that $M=(M, g)$ is an $n$ dimensional Riemannian manifold whose unit tangent sphere bundle $T_{1} M$ (equipped with the standard contact metric structure $(\eta, \bar{g}, \phi, \xi))$ is H-contact, unless otherwise specified. Then, since the characteristic vector field $\xi$ is an eigenvector field of the Ricci operator $\bar{Q}$ of $T_{1} M$, there exists a smooth function $\bar{\lambda}$ (call it the corresponding eigenvalues of $\bar{Q}$ ) satisfying

$$
\bar{Q} \xi=\bar{\lambda} \xi .
$$


Thus, from (2.1), (4.3), (4.9) and (5.3), we have

$$
\begin{aligned}
\bar{\lambda} & =4 \rho(u, u)-2 \alpha(u, u) \\
& =-4 \rho(u, u)+\frac{8}{n} \tau-\frac{2|\rho|^{2}}{n(n+2)}-\frac{3|R|^{2}}{n(n+2)}
\end{aligned}
$$

on $T_{1} M$.

Further, from (2.1), we also see that the scalar curvature $\bar{\tau}$ of $T_{1} M$ is given by

$$
\bar{\tau}=4(n-1)(n-2)+4 \tau-\alpha(u, u)
$$

for any $u \in T_{1} M$. Now, we shall prove the following:

Theorem 2. Let $M=(M, g)$ be an $n(\neq 4)$-dimensional Riemannian manifold whose unit tangent sphere bundle $T_{1} M$ is $H$-contact. Then, the followings are equivalent:

(1) the corresponding eigenvalue $\bar{\lambda}$ of the Ricci operator $\bar{Q}$ of $T_{1} M$ is constant.

(2) the scalar curvature $\bar{\tau}$ of $T_{1} M$ is constant.

(3) $M$ is 2-stein.

Proof. It suffices to prove the equivalence of (1) and (3) and the equivalence of (2) and (3). Since $\operatorname{dim} M \neq 4$, from (5.4), taking account of Main Theorem and the result ([8]), we have the equivalence of (1) and (3).

Similarly, from (5.1), (5.5) and Main Theorem, we see easily that $\bar{\tau}$ is constant on $T_{1} M$ if and only if the equality (5.2) holds on $M$. Thus, from (4.9) and $(4.14) \sim(4.16)$, taking account of Main Theorem, we have

$$
\underset{b, c, d}{\mathfrak{S}} \nabla_{b} \rho_{c d}=0
$$

Thus, from (3.1) and (5.6), it follows immediately that $\nabla \rho=0$. By the result of Calvaruso and Perrone ([5], Theorem 4.2), we see that $M$ is Einstein (and hence, 2-stein). This completes the proof of the equivalence of (2) and (3).

Now, let $M=(M, g)$ be a locally symmetric space whose unit tangent sphere bundle $T_{1} M$ is H-contact. Then, we also see that $M$ is Einstein (and hence, 2-stein). Thus, in this case, we see that the corresponding eigenvalue $\bar{\lambda}$ of the Ricci operator $\bar{Q}$ of $T_{1} M$ and the scalar curvature of the unit tangent sphere bundle $T_{1} M$ of $M$ are both constant by virtue of Theorem 2 . So, in order to determine the base manifold whose unit tangent sphere bundle $T_{1} M$ is $\mathrm{H}$ contact, it seems reasonable to start with the case where the scalar curvature of $T_{1} M$ is constant. Therefore, as a special case of Question 1, the following question will be raised.

Question 2. Is $n(\neq 4)$-dimensional 2-stein manifold locally symmetric? 
Acknowledgements. Research of S. H. Chun was supported by the National Research Foundation of Korea Grant funded by the Korean Government[NRF2009-351-C00010], Research of H. K. Pak and J. H. Park was supported by Basic Science Research Program through the National Research Foundation of Korea(NRF) funded by the Ministry of Education, Science and Technology(20090087201).

\section{References}

[1] A. L. Besse, Manifolds All of Whose Geodesics are Closed, Springer-Verlag, Berlin-New York, 1978.

[2] D. E. Blair, Riemannian Geometry of Contact and Symplectic Manifolds, Birkhäuser, Boston, Inc., Boston, MA, 2002.

[3] E. Boeckx and L. Vanhecke, Harmonic and minimal vector fields on tangent and unit tangent bundles, Differential Geom. Appl. 13 (2000), no. 1, 77-93.

[4] _ Unit tangent sphere bundles with constant scalar curvature, Czechoslovak Math. J. 51(126) (2001), no. 3, 523-544.

[5] G. Calvaruso and D. Perrone, H-contact unit tangent sphere bundles, Rocky Mountain J. Math. 37 (2007), no. 5, 1435-1458.

[6] P. Carpenter, A. Gray, and T. J. Willmore, The curvature of Einstein symmetric spaces, Quart. J. Math. Oxford Ser. (2) 33 (1982), no. 129, 45-64.

[7] Y. D. Chai, S. H. Chun, J. H. Park, and K. Sekigawa, Remarks on $\eta$-Einstein unit tangent bundles, Monatsh. Math. 155 (2008), no. 1, 31-42.

[8] S. H. Chun, J. H. Park, and K. Sekigawa, H-contact unit tangent sphere bundles of Einstein manifolds, Quart. J. Math., (to appear), DOI:10.1093/qmath/hap025.

[9] P. Nurowski and M. Pruzanowski, A four-dimensional example of a Ricci flat metric admitting almost-Kähler non-Kähler structure, Classical Quantum Gravity 16 (1999), no. 3, L9-L13.

[10] T. Oguro, K. Sekigawa, and A. Yamada, Four-dimensional almost Kahler Einstein and weakly *-Einstein manifolds, Yokohama Math. J. 47 (1999), no. 1, 75-92.

[11] J. H. Park and K. Sekigawa, When are the tangent sphere bundles of a Riemannian manifold $\eta$-Einstein?, Ann. Global Anal. Geom. 36 (2009), no. 3, 275-284.

[12] _ Notes on tangent sphere bundles of constant radii, J. Korean Math. Soc. 46 (2009), no. 6, 1255-1265.

[13] D. Perrone, Contact metric manifolds whose characteristic vector field is a harmonic vector field, Differential Geom. Appl. 20 (2004), no. 3, 367-378.

[14] C. M. Wood, On the energy of a unit vector field, Geom. Dedicata 64 (1997), no. 3, 319-330.

Sun Hyang CHun

Department of Mathematics

SUNGKYUNKWAN UNIVERSITY

SuwON 440-746, KoreA

E-mail address: cshyang@skku.edu

HONG KYUNG PAK

FACUlty of Information and Science

DAEGU HAANY UNIVERSITY

KyUnGSAN 712-715, KorEA

E-mail address: hkpak@dhu.ac.kr 
JEONG HYEONG PARK

Department of Mathematics

SUNGKYUNKWAN UNIVERSITY

Suwon 440-746, Korea

E-mail address: parkj@skku.edu

Kouei Sekigawa

Department of Mathematics

NiIgATA University

NiIGATA 950-2181, JAPAN

E-mail address: sekigawa@math.sc.niigata-u.ac.jp 